\title{
B Cell Lymphoma of Thoracic Vertebrae: A Great Mimicker
}

\author{
Wan Irfan Wan Mustapha \\ Department of Radiology, International Islamic University Malaysia Medical Centre.
}

Introduction: Non Hodgkin lymphoma (NHL) is a malignant neoplasm affecting multiple systems in which extranodal NHL represents $10-20 \%$ of all NHL. Case report: This is a case of 45-year-old female with no known medical illness who presented with progressive numbness of bilateral lower limbs since March 2016 which subsequently progress to loss of motor power since November 2016. She initially was diagnosed as tuberculous (TB) spondylitis which then turned out to be $B$ cell lymphoma of the thoracic vertebra. The diagnosis was confirmed on histopatological examination (HPE) after patient underwent laminectomy of T3-T7 vertebra bodies and excision biopsy with removal of the casseous material. Due to a lack of specific findings, the diagnosis of vertebral NHL is often missed or delayed. It warrants attention that NHL of thoracic vertebra may be misdiagnosed as TB spondylitis due to their similarities and sometimes the imaging features may simulate and overlap each other. The main treatment for vertebral NHL is chemotherapy, radiotherapy or both. Surgery maybe necessary if patient has story of neurological deficits. However patient was treated conservatively as she refused chemotherapy. 